Research Article

\title{
Hybrid Numerical Simulation of Jet Blast Distance of a Departing Aircraft
}

\author{
Xin He, ${ }^{1}$ Yaqing Chen $\left(\mathbb{D},{ }^{2}\right.$ Yilong $M a,{ }^{1}$ Dengfeng $\mathrm{Hu},{ }^{1}$ and Haoran Gao ${ }^{3}{ }^{3}$ \\ ${ }^{1}$ Air Traffic Management College, Civil Aviation Flight University of China, Guanghan 618307, China \\ ${ }^{2}$ CAAC Key Laboratory of Flight Technology and Safety, Civil Aviation Flight University of China, Guanghan 618307, China \\ ${ }^{3}$ Institute Office, Civil Aviation Flight University of China, Guanghan 618307, China
}

Correspondence should be addressed to Yaqing Chen; chenyaqingmail@sina.com

Received 2 February 2021; Revised 1 March 2021; Accepted 14 March 2021; Published 31 March 2021

Academic Editor: Mohammad Yazdi

Copyright $\odot 2021$ Xin He et al. This is an open access article distributed under the Creative Commons Attribution License, which permits unrestricted use, distribution, and reproduction in any medium, provided the original work is properly cited.

\begin{abstract}
A hybrid numerical simulation method was established by combining the Spalart-Allmaras (SA) turbulence model and detached eddy simulation (DES). Numerical simulations were carried out to model cold and hot spray conditions of a nozzle without considering the internal flow of an engine to determine jet conditions. Analysis results show that the calculated hot spray results more in line with the reality. The jet effect of a typical aircraft engine was simulated numerically to determine the distance influenced by the jet blast from a departing aircraft engine.
\end{abstract}

\section{Introduction}

To address the strains created by an increasing number of flights and the lack of airport support capability, major airports in China have been reconstructed and expanded. At present, more than ten airports in China have adopted a multiple runway operation mode [1-3]. Most of China's multiple runway airports have adopted a parallel runway mode, making it difficult to avoid the problem of runway crossings during actual operation. China has the following runway-crossing protocols. First, "if one aircraft enters the runway, then other aircraft cannot cross the runway," such as at Shanghai Hongqiao International Airport and Shanghai Pudong International Airport. Second, "one can choose to cross the runway from the front side of a departing aircraft," such as at Chongqing Jiangbei International Airport. Third, "bypass the runway in a U-shaped path to achieve the purpose of crossing," such as at Beijing Capital Airport. The above methods for crossing a runway avoid threatening aircraft safety during ground operations but seriously restrict operational efficiency of the ground. Therefore, China has considered an operational mode that traverses the runway from the rear side of the departing aircraft, taxi-behind, as shown in Figure 1.
The mode taxi-behind is to cross the runway behind the departing aircraft in order to reduce the operational conflict of the airport scene and improve operational efficiency, for example, $\mathrm{O}$ 'Hare Airport in the United States has used this model and demonstrated good safety and efficiency improvements.

The jet blast of the departing aircraft is the most important factor hindering the implementation of taxi-behind. Without adequate safety interval, an aircraft crossing a runway could be blown off course or even overturned by the strong aerodynamic load of the jet blast from the departing aircraft. The magnitude of the safety interval mainly depends on the strength of the jet blast of the departing aircraft and the bearing capacity of the runway-crossing aircraft, so it is necessary to study the influence distance of the jet blast of the departing aircraft engine. At present, computational fluid dynamics (CFD) numerical simulation and the real engine test are the main methods to analyze the impact of aircraft jet blast. Due to the difficulty, high cost, and great safety risk of the real engine test on the runway and aircraft engine data are highly confidential in countries, we can only get the limited data of jet velocity distribution from the engine manufacturer or the aircraft manufacturer, and it is not enough to be used in the research of the runway 


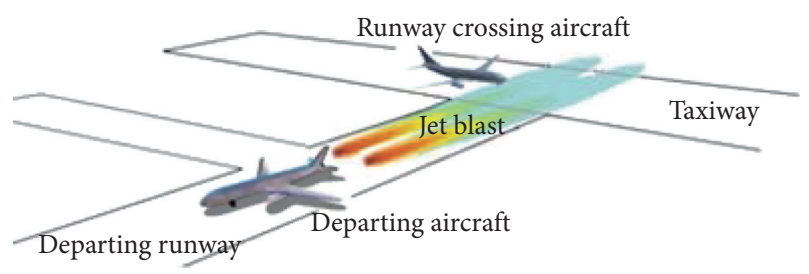

FIgUre 1: The taxi-behind mode schematic diagram.

operation mode. The hybrid numerical simulation method was adopted in this study for research.

Compared with domestic research, foreign research in this field was carried out earlier. In addition to the experimental method, the numerical simulation method was used to obtain the formation and evolution process of the jet as well as the parameters such as velocity, pressure, and temperature [4]. The influence of jet on landing aircraft is also analyzed in some literatures [5]. In 2006, MelberWilkending [6] established and verified the process chain based on the adaptive DLR TAU program, using CFD considering as example a Hangar and an AIRBUS A380 configuration, and gave the flow solver program for determining the jet wake of the rear engine. Slaboch in Volpe National Transportation Systems Center [7] presents an operational model for the prediction of jet blast, and the model was developed based upon three modules including a jet exhaust model, jet centerline decay model, and aircraft motion model. The final analysis was compared with data acquired during a jet blast study conducted by the Volpe Center in 1999. This analysis closely matched the data from the 1999 test. The analysis was then used to predict the jet blast evolution from aircraft that have been introduced since 1999, including the Boeing 777-300ER. Ishiko et al. [8] in order to examine the far-field velocity of jet blast by computational fluid dynamics (CFD) in this research, simulation using Reynolds-averaged Navier-Stokes (RANS) equation is carried out with very large computational domain. The velocity profiles obtained by numerical simulation agree well with those obtained by experiments. Then, they performed the parametric study of wind direction. It is found that the wind direction angle that has the most effect on the deflection of jet blast is lower than ninety degrees. Supported by theoretical and wind tunnel modeling, Bennett et al. [9] have experimented with an array of aerodynamic baffles on the surface behind a set of turbofan engines of $124 \mathrm{kN}$ thrust. Lidar and point sampler measurements show that, as long as the intervention takes place within the zone where the Coanda effect holds the jet to the surface (i.e., within about $70 \mathrm{~m}$ in this case), quite modest surface-mounted baffles can rapidly lift the jet away from the ground. This was of potential benefit in abating both surface concentrations and jet blast downstream. There were also some modest acoustic benefits. The three-dimensional jet flow field of aircraft was simulated by Taguchi et al. [10]; they used a supersonic nozzle wall jet device, and the experimental results were compared with the calculation results of the SST turbulence model. The results show that the existing Reynolds stress database is of great significance to the establishment of the wall jet turbulence model. In recent decades, the RANS
(Reynolds-averaged Navier-Stokes) method has been used to study jet flow fields in aviation. However, reliable numerical results have not been obtained [11]. Although the RANS method requires the minimal computational effort, the pulsating motion details are smoothed out by homogenizing the turbulent fluctuations [12]. Therefore, it is impossible to accurately simulate the growth rate of the shear layer and turbulence details such as the inviscid velocity of the jet, which is not affected by the shear layer [13-15]. Scholars at home and abroad have used the LES (large eddy simulation) method to carry out numerical simulations on jet blast. The basic concept has been to directly and numerically simulate large eddies by using the spatial sieve filtration method, Fourier function, or Gaussian function to separate large and small eddies [16-18]. The subgrid stress model has been used to calculate small eddies [19]. In [20], the LES method was used to simulate the aerodynamic noise and coaxial twin jet flow noise before and after installing a zigzag nozzle, and the effect of the zigzag nozzle on the flow field structure and sound field was studied. In [21], free flow and jet flow numerical simulations were carried out with the LES method. The results showed that the location and shape of large turbulence structures were consistent with the experimental images. Although the LES method has achieved good results, in an actual numerical simulation process, even the LES method needs a considerable grid support to capture small eddies near the engine walls [22]. Moreover, when assessing the complex flow of engine jets, the near-wall mode of the LES method cannot accurately separate the high Reynolds number near-wall turbulence, so it can only deal with low Reynolds number turbulent flows [23, 24].

At present, the DES (detached eddy simulation) method has been introduced to assist in the numerical simulation of jet engines. It is a numerical method used for the calculation of detached vortices $[25,26]$. The basic concept employs the RANS method to simulate small-scale turbulent fluctuations near the engine wall. In the area far away from the boundary layer, a Smagorinsky large eddy simulation is used to simulate the detached vortex to enable the subgrid Reynolds stress model [27]. This method has two advantages. First, it avoids the problem of a larger grid size in the conventional boundary layer method (LES). The method also ensures that the large-scale detached vortex energy can be accurately simulated in a range far away from the surface of the object. Liu et al. [28] used a mixed unstructured grid and an SST turbulence model to simulate a jet engine with the DES method and compared it with experimental results. The rationality of the method was proven. Dietiker and Hoffmann [29] used the DES method to study a three-dimensional unsteady jet and compared it with experimental data to obtain relatively good numerical results. Gong et al. [30] proposed an improved delay separation vortex simulation (IDDES) method based on the SST model for a high-precision simulation of the jet engine effect and conducted a numerical study of the dynamic jet effect through a RANS/ LES hybrid method. Their results showed that the velocity distribution in a jet blast can be predicted better [31, 32].

In summary, DES offers great advantages in the numerical simulation of jet blast, but domestic scholars have 
seldom used this approach. At present, domestic and foreign scholars have performed more numerical simulations of near-field jet blasts [33-37]. However, for taxiing behind of the departing aircraft, more attention has been paid to the far-field flow of the jet [38-40]. The SA turbulence model, as a classic aviation field model, exhibits strong robustness and a rich experience value [41, 42]. Therefore, based on the SA turbulence model and the DES method, a DES-SA mixed numerical simulation method is built to simulate the far field of a jet blast. A nozzle model that does not consider internal engine flow is used. Two different jet states from a Boeing aircraft nozzle are calculated, and the calculated state, which is more consistent with the working conditions of the engine, is determined. On this basis, the influence distance of a typical jet blast is studied to provide the necessary theoretical and technical support for the implementation of a taxibehind mode. At the same time, it provides a reference for the study of engine jet by the DES numerical simulation method.

\section{Methodology}

$\mathrm{N}-\mathrm{S}$ equations are the most complete governing equations describing fluid motion so far and can be written in the form of Cartesian tensor using summation convention.

The continuity equation is

$$
\frac{\partial \rho}{\partial t}+\frac{\partial}{\partial x_{i}}\left(\rho u_{i}\right)=0 .
$$

The momentum equation is

$$
\frac{\partial}{\partial t}\left(\rho u_{i}\right)+\frac{\partial}{\partial x_{i}}\left(\rho u_{j} u_{i}\right)=-\frac{\partial p}{\partial x_{i}}+\frac{\partial \sigma_{i j}}{\partial x_{j}}+\frac{\partial}{\partial x_{j}}\left(-\rho u_{j}^{\prime} u_{i}^{\prime}\right),
$$

where $u_{i}$ is the Reynolds average velocity component omitting the average sign; $\rho$ is the density; $p$ is the pressure; $u_{i}^{\prime}$ is the pulsating velocity; $\sigma_{i j}$ is the stress tensor component.

Based on the SA turbulence model combining the DES method, the hybrid DES-SA numerical simulation method is used to simulate the far-field flow of a jet engine $[43,44]$. The integral method for the DES-SA approach is as follows:

$$
\frac{\partial}{\partial t} \int_{\Omega} \widetilde{v} \mathrm{~d} \Omega+\oint_{\partial \Omega}\left(F_{c, T}-F_{v, T}\right) \mathrm{d} S=\int_{\Omega} Q_{T} \mathrm{~d} \Omega,
$$

where $\widetilde{v}$ is the viscosity coefficient; $F_{c, T}$ is the convective flux term; $F_{v, T}$ is the viscous flux term; $Q_{T}$ is the source item which contains generation and destruction items.

The mixed model adopts a mixed length scale $l_{\text {DES }}$ instead of the traditional SA turbulence model $d$ to calculate the distance from the unit to the nearest wall; the following formula is adopted:

$$
\tilde{d}=\min \left(d, C_{\mathrm{DES}} \Delta\right),
$$

where $C_{\mathrm{DES}}$ is the adjustable coefficient, which is usually $0.65 ; \Delta$ is the filter scale in the LES method.

Therefore, the DES-SA mixing method has $d \ll \Delta\left(l_{\mathrm{DES}}=\right.$ $d$ ) in the near-wall region. The traditional SA turbulence model is used for numerical simulation, which has $d \gg \Delta\left(l_{\mathrm{DES}}=\Delta\right)$ far away from the wall area. When there is a balance between the production term and the dissipation term, $\tilde{v} \propto S \Delta^{2}$. At this point, the DES-SA mixing method is transformed into the LES method to simulate the jet flow.

At present, the main method for nozzle boundary condition construction depends on whether to consider the internal flow of the nozzle. For the case of internal nozzle flow, the boundary of the computational domain lies within the nozzle. The boundary conditions are usually set as the total pressure and total temperature inside the nozzle or the total pressure ratio of the internal combustion chamber (the combustion chamber) to the external atmosphere $\left(P_{0} / P\right)$ and total temperature ratio $\left(T_{0} / T\right)$ [45]. This method restricts the size and internal configuration of nozzle. It can fully consider the viscous effect of the inner wall in the engine nozzle [46]. The calculation accuracy is relatively high. However, considering that the nozzle is a highly proprietary product, the internal structure and size data are difficult to obtain. Therefore, the internal flow of the nozzle is not considered in this study. We only require the Mach number, static pressure, and static temperature at the outlet of the nozzle and regard the nozzle outlet condition as the entry condition for the solution domain of the flow field.

\section{Example Verification Analysis}

Considering the complex working state of the engine, scholars at home and abroad typically compare the two states of cold and hot spray when calculating a jet engine field and analyze the similarities and differences between the two states of cold and hot spray. Then, according to the actual situation, the conditions that are more suitable for the actual working conditions are determined for numerical simulation. In this study, the combined DES-SA method is used to study the two states of engine cold jetting and hot spraying. The setting of specific parameters is given in Table 1.

Taking a Boeing A320 aircraft (engine type is CFM56$5 \mathrm{~B})$ as an example, according to the actual engine size, the twin nozzle model in the calculation example is scaled down. The diameter of the CFM56-5B engine fan nozzle is $1.735 \mathrm{~m}$; the specific structure is shown in Figures 2 and 3.

To investigate the ground effect, the calculation domain is chosen as follows: according to the assembly height of the A320 aircraft engine (the vertical height of the axis from the ground is approximately $1.68 \mathrm{~m}$ ), the height distance from the ground is $1.7348 \mathrm{~m}$ and perpendicular to the direction of the incoming flow. The width of the flow field is $45.533 \mathrm{~m}$, and the height is $24.5013 \mathrm{~m}$ along the streamline direction. The length of the flow field is $341.498 \mathrm{~m}$. To obtain more accurate calculation results, the boundary conditions are set as follows: the ground is set as the boundary condition of the material surface, the outlet is set as the pressure outlet condition, and the three planes and the flow inlet surface parallel to the flow are set as the far-field pressure condition. The calculation domain size is shown in Figure 4.

During mesh processing, the mesh at the nozzle outlet is encrypted to better simulate the velocity change at the nozzle outlet, as shown in Figures 5(a) and 5(b). During the volume 
TABLE 1: Calculation state of double-channel jet flow.

\begin{tabular}{llccrc}
\hline Case & $M_{\mathrm{pj}}$ & $M_{\mathrm{sj}}$ & $\mathrm{TTR}_{p}$ & $\mathrm{TTR}_{s}$ & \\
I & 0.85 & 0.95 & 1.0 & 1.0 & $M_{\mathrm{cf}}$ \\
II & 0.85 & 0.95 & 2.26 & 1.0 & 0.05 \\
\hline
\end{tabular}

$M_{\mathrm{pj}}$, the Mach number of the inner nozzle outlet; $M_{\mathrm{sj}}$, the fan outlet Mach number; TTR, the total temperature ratio of the inner and outer flow fields of the nozzle; $M_{\mathrm{cf}}$, the number of incoming Mach flows for the external flow field of the nozzle.

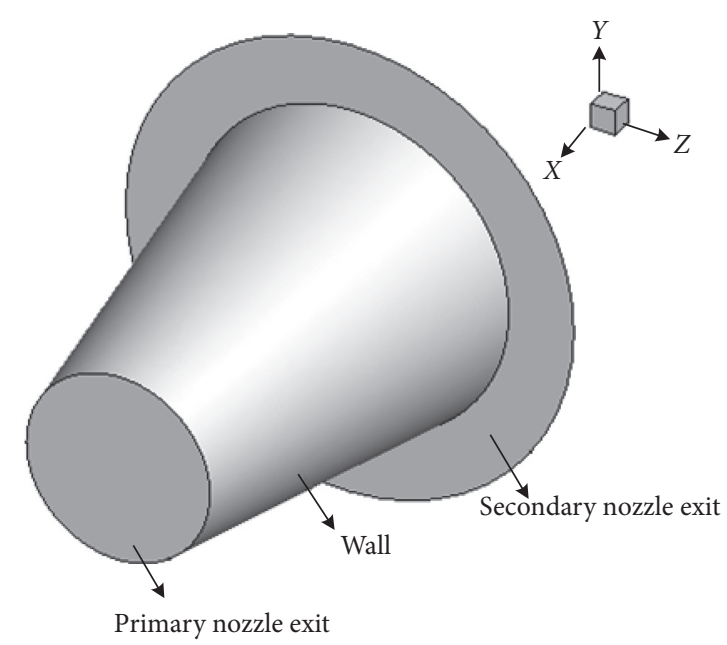

Figure 2: Three-dimensional structure of the nozzle ( $x$, axial direction; $y$, radial direction; $z$, height direction).

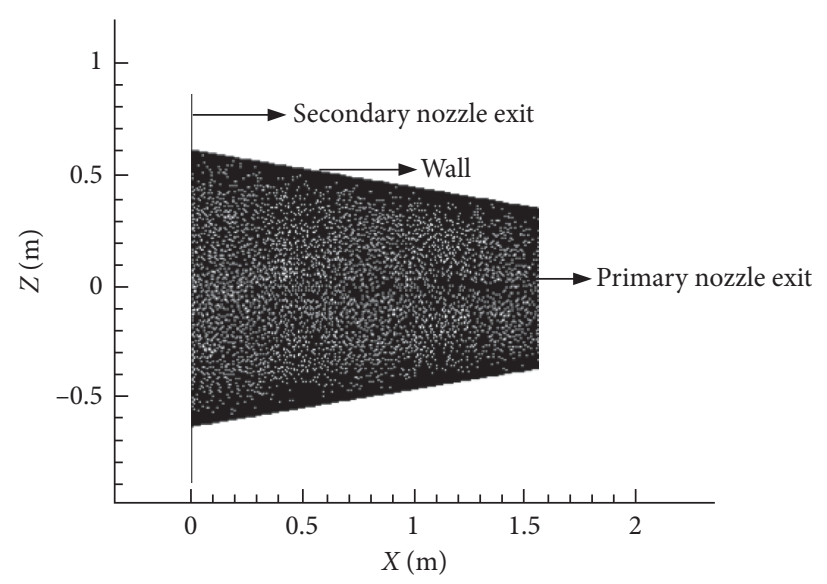

Figure 3: Top view of the nozzle structure.

mesh generation, in order to take the ground effect into account, the mesh at the ground and the nozzle is encrypted, as shown in Figures 5(c) and 5(d). The specific number of grid nodes and cells is shown in Table 2

The boundary conditions of the external flow field are as follows: static pressure $P=101325 \mathrm{~Pa}$, static temperature $T=298 \mathrm{~K}$, and working gas is the ideal gas. According to formula (5) and the total temperature ratio, the static temperature and static pressure at the outlet of the inner nozzle and outer nozzle can be determined.

$$
\frac{T_{0}}{T}=\left(1+\frac{\gamma-1}{2} M^{2}\right)
$$

Figures 6 and 7 show the results of the numerical simulation of the nozzle axis velocity varied with the nozzle distance obtained from the numerical simulation. In the graph, we can see that the axial velocity of the nozzle decreases rapidly within the distance from the front $100 \mathrm{~m}$; regardless of whether the nozzle is heated or not, the velocity slows down from $100 \mathrm{~m}$, and the axis velocity at $300 \mathrm{~m}$ is still approximately $60 \mathrm{~m} / \mathrm{s}$. Compared with the incoming velocity $(16.6 \mathrm{~m} / \mathrm{s})$, the speed is still greater. There is a risk in crossing the runway at this distance. Therefore, it can be concluded that the static temperature at the outlet of the nozzle has a little effect on the speed drop in the range from $100 \mathrm{~m}$ to $300 \mathrm{~m}$ downstream. 


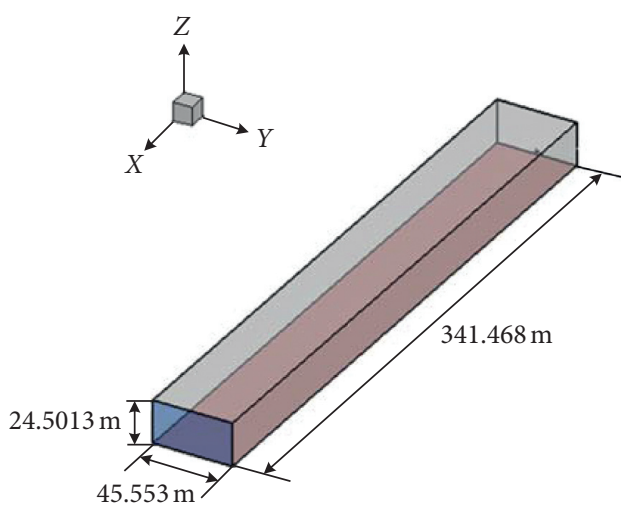

(a)

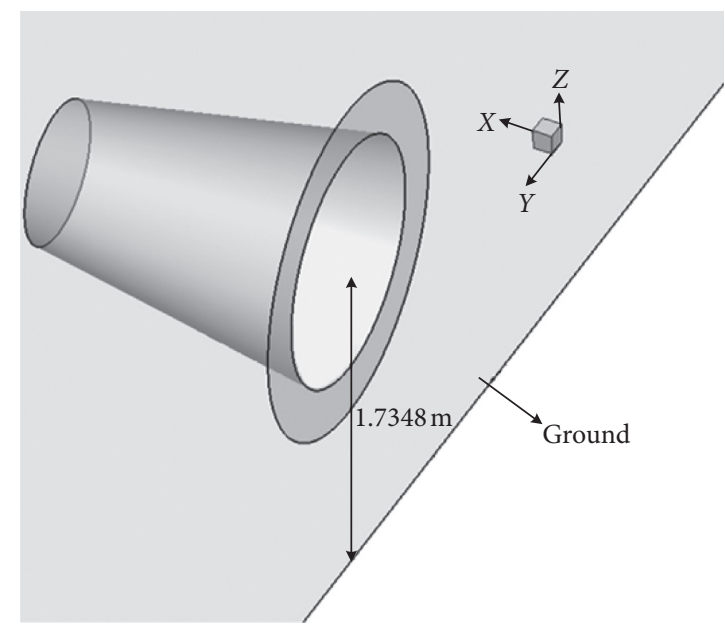

(b)

FIGURE 4: Calculation domain size ( $x$, flow direction; $y$, direction; $z$, height direction).

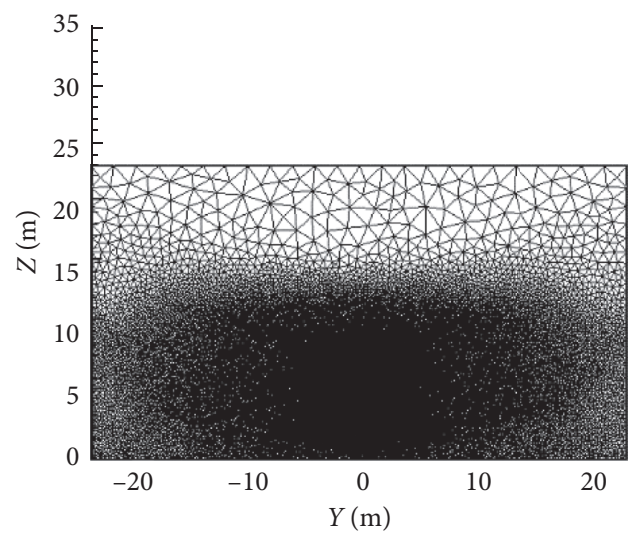

(a)

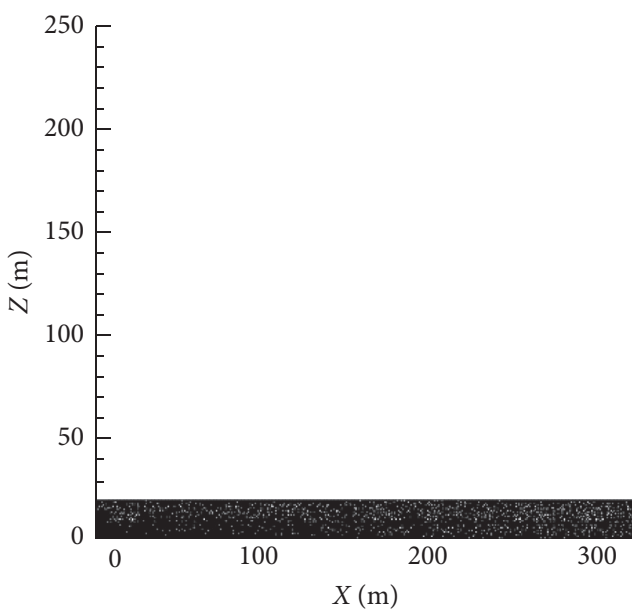

(c)

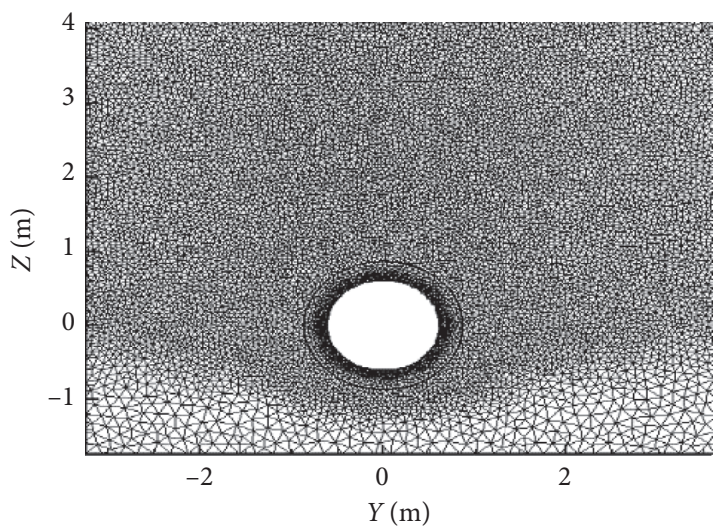

(b)

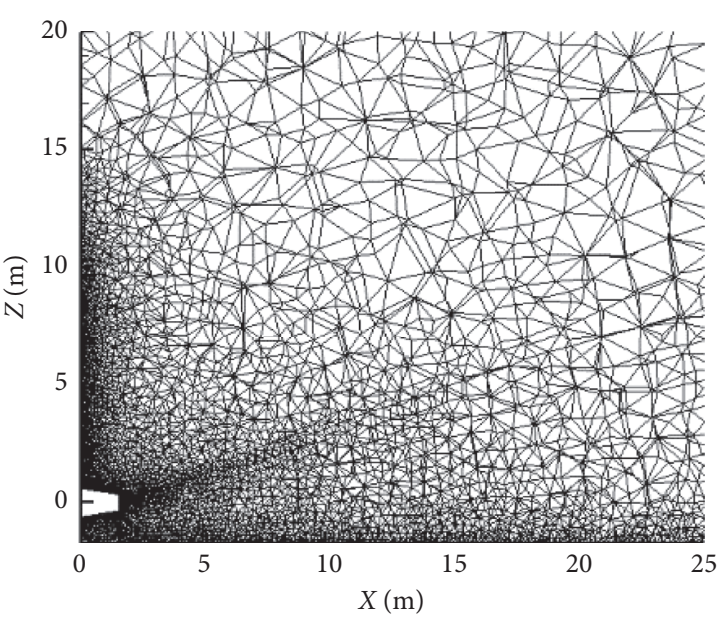

(d)

Figure 5: Grid details. (a) Nozzle outlet surface mesh. (b) Local surface mesh at nozzle outlet. (c) The symmetry plane of the flow field is the volume grid. (d) Section of the volume mesh at the nozzle exit.

Figures 8 and 9 show the velocity clouds of the nozzle outlet under cold spray and hot spray conditions, respectively. Figure 8 shows that the velocity at the outlet of the nozzle is the greatest. As the distance increases, the velocity decreases gradually due to the dissipation of energy, and the incoming gas flow also creates a shearing action with the tail 
TABLE 2: Number of mesh nodes and mesh cells.

\begin{tabular}{ll}
\hline Grid nodes & Grid cell \\
942,325 & $5,131,569$
\end{tabular}

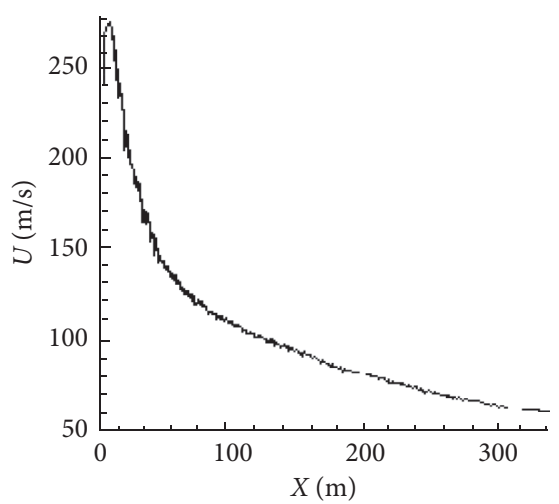

FIgURE 6: Axis velocity of the nozzle axis at $\operatorname{TTR}_{p}=1.0$.

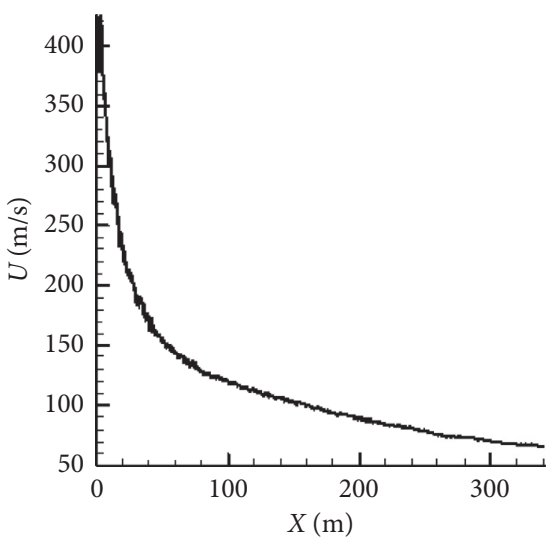

Figure 7: Axis velocity of the nozzle axis at $\operatorname{TTR}_{p}=2.26$.

jet stream at high speed, resulting in an increase in the gas flow velocity near the tail jet stream. It can be seen in Figure 9 that although the Mach number at the outlet is less than the Mach number at the culvert, the speed at the outlet is faster than that at the culvert. The reason is that the total temperature at the outlet is higher and the static temperature is higher than the static temperature at the culvert, so the corresponding sound speed is also faster. Although the Mach number is relatively low, the absolute velocity of the calculated airflow is still faster.

Figures 10 and 11 show the temperature cloud map at the outlet of the nozzle calculated under cold spray and hot spray conditions, respectively. In the figures, we can see that the influence range of the tail jet increases with increasing distance. The absolute value of the difference between the center and the ambient temperature decreases with increasing distance. However, the total temperature ratio is 1 under the cold spray condition, and the gas energy inside and outside the nozzle is the same. According to formula (5), when the total temperature is constant, the larger the Mach number is, the lower the static temperature is. Therefore, the static temperature at the outlet of the nozzle is lower than that of the outside atmosphere. It is obvious that this example is different from an actual engine situation.

During the heating state, the static temperature of the outlet is higher than the ambient temperature, while the static temperature of the outlet is lower than the ambient temperature. This is due to the heating at the outlets and the higher total gas temperature at the outlets. At a certain Mach number, the static temperature at the outlet of the inner nozzle is far higher than that of the environment. However, the Mach number is larger than the external atmosphere, so the static temperature at the outlet is lower. It is worth noting that the static temperature at the center of the tail gas jet is higher than that of the external atmosphere compared with the cold spray state, which is also due to the higher static temperature of the gas in the cones. From the above analysis, the hot injection state is more consistent with the real working situation of the engine.

\section{Numerical Simulation Analysis of Typical Jet Engine Flow}

The influence distance of a departing aircraft's jet blast is very important for determining the safe clearance of the rear crossing mode. Therefore, this section performs a numerical simulation analysis on the jet effect of a typical engine. According to CAAC data, as of February 2019, the proportion of Airbus and Boeing aircrafts in domestic airlines is 94.66\%. Among them, the A320, B777, and B737-800 account for up to $78.18 \%$ of the total. The CRJ900, as a typical representative of the regional aircraft, is favored for its excellent flight performance and low fuel consumption. Therefore, the above four models are selected and categorized according to their wake ratings: light-duty (CRJ900), medium-duty (A320 and B737-800), and heavy-duty (B777). The thermal jet method is used to analyze and calculate the influence distance of the jet blast.

(1) Determine the shrinkage of the calculation model When calculating the far-field tail jet flow due to the lack of actual size data, the model is scaled according to the actual engine's fan diameter

(2) Calculate the true flow of internal and external channels

The air flow in the ducts is calculated according to the bypass ratio and the total air flow rate. The formula is as follows:

$$
\begin{aligned}
\dot{m}_{\text {pilot_duct }} & =\frac{\dot{m}_{\text {total }}}{(\text { bypass_ratio }+1)}, \\
\dot{m}_{\text {external_duct }} & =\dot{m}_{\text {total }}-\dot{m}_{\text {pilot duct }} .
\end{aligned}
$$

(3) Calculate the speed of internal and external channels For a turbofan engine, the ratio of internal and external ducts is $2: 8$. Therefore, the velocity of the internal and external ducts can be calculated according to the momentum theorem under the 


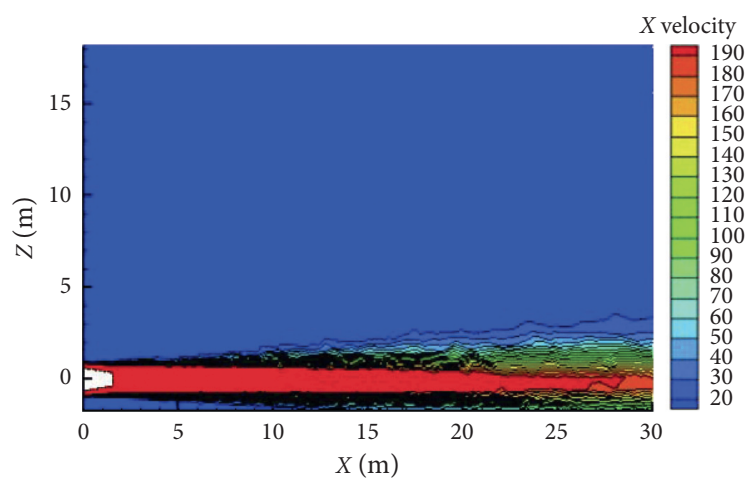

Figure 8: Velocity cloud map of the nozzle outlet at $\mathrm{TTR}_{p}=1.0$.

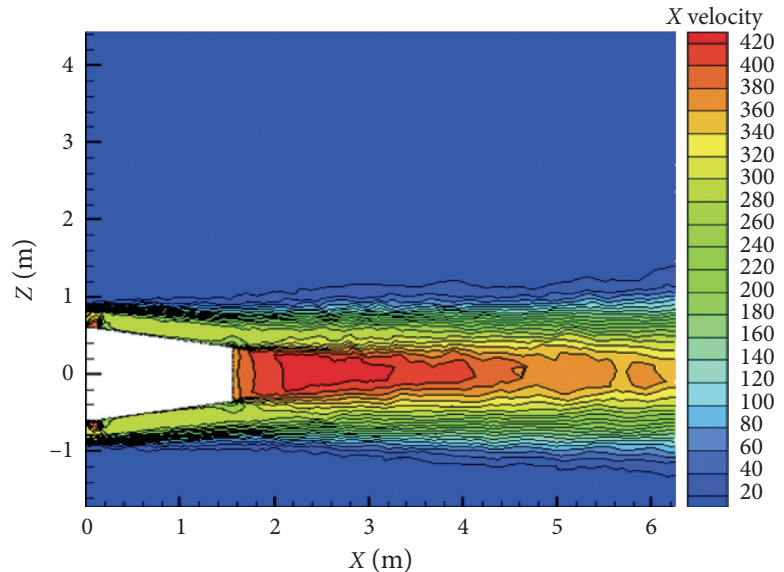

FIgURE 9: Velocity cloud map of the nozzle outlet at $\operatorname{TTR}_{p}=2.26$.

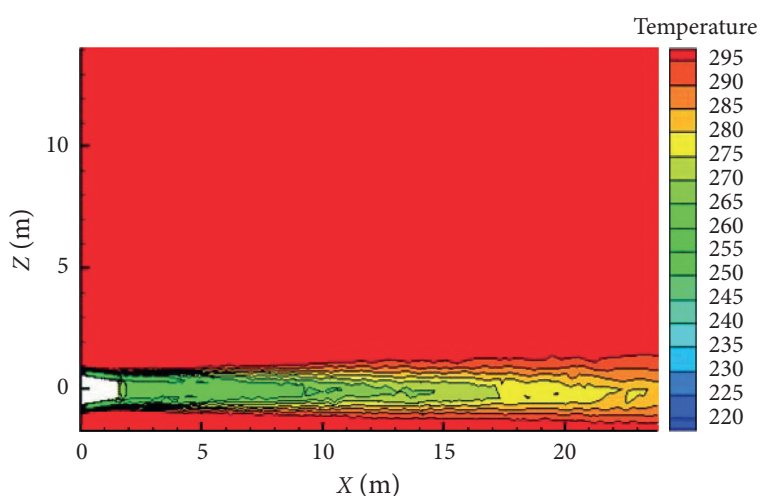

Figure 10: Temperature cloud map of the nozzle outlet at $\mathrm{TTR}_{p}=1.0$.

condition of known internal and external channel flows.

$$
F \Delta t=\dot{m} \Delta V
$$

(4) Determine the Mach numbers inside and outside the culvert

To determine the speed and temperature inside and outside the culvert, the Mach number of the inner and outer ducts can be calculated according to the following formulas.

$$
\begin{aligned}
a & =\sqrt{\gamma R T}, \\
M a & =\frac{V}{a} .
\end{aligned}
$$

Taking A320 as an example, the real jet blast tail effect is calculated. The A320 mainly uses the CFM56-5B engine, and the relevant parameters are given in Table 3.

According to formulas (6)-(10), the boundary conditions at the nozzle outlet can be obtained, as given in Table 4 .

Through numerical simulations of the above cases, the jet velocity distribution curve and the velocity profile of the jet $X Z$ section are obtained (Figures 12 and 13). According to the limitations in the Flight Crew Operation Manual (FCOM), this study mainly analyzes jet engines at speeds of $44 \mathrm{~m} / \mathrm{s}, 22 \mathrm{~m} / \mathrm{s}$, and $16 \mathrm{~m} / \mathrm{s}$. Figure 13 shows that the influence distances of A320 jet blast at speeds of $44 \mathrm{~m} / \mathrm{s}$, $22 \mathrm{~m} / \mathrm{s}$, and $16 \mathrm{~m} / \mathrm{s}$ are $90 \mathrm{~m}, 296 \mathrm{~m}$, and $451 \mathrm{~m}$, respectively.

Similarly, numerical simulations for the B777, B737-800, and CRJ900 are carried out to obtain the velocity distributions of their jet blasts, as shown in Figures 14-16. 


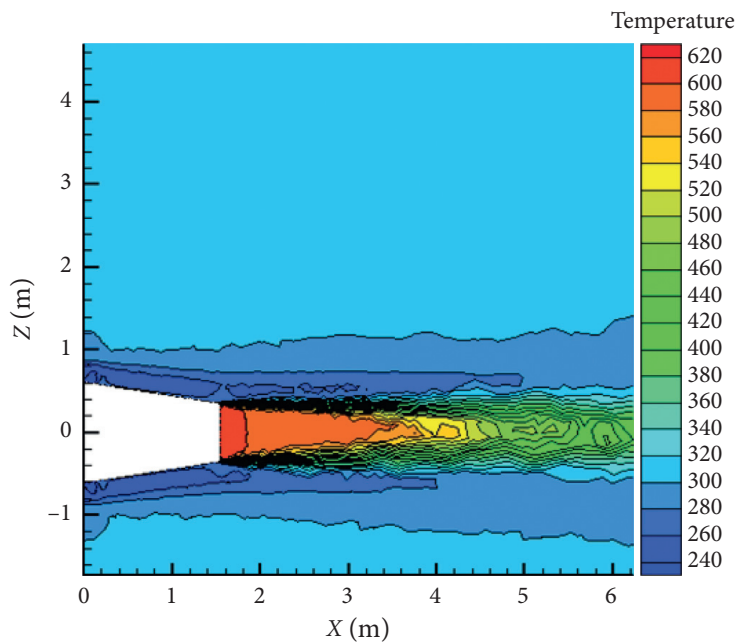

Figure 11: Temperature cloud map of the nozzle outlet at $\operatorname{TTR}_{p}=2.26$.

TABle 3: A320 engine parameters.

Maximum take-off thrust $(\mathrm{daN}) \quad$ Air mass flow $(\mathrm{kg} / \mathrm{s}) \quad$ Bypass ratio $\quad$ Diameter $(\mathrm{mm}) \quad$ Engine outlet temperature $\left({ }^{\circ} \mathrm{C}\right)$
13360 390.5 5.0 1735 297

TABle 4: Dynamic boundary conditions of the A320 engine.

\begin{tabular}{lccccc}
\hline $\begin{array}{l}\text { Internal nozzle static } \\
\text { pressure }(\mathrm{Pa})\end{array}$ & $\begin{array}{c}\text { Internal nozzle static } \\
\text { temperature }(\mathrm{K})\end{array}$ & $\begin{array}{c}\text { Mach number of } \\
\text { internal nozzle }\end{array}$ & $\begin{array}{c}\text { External nozzle } \\
\text { static pressure }(\mathrm{Pa})\end{array}$ & $\begin{array}{c}\text { External nozzle static } \\
\text { temperature }(\mathrm{K})\end{array}$ & $\begin{array}{c}\text { Mach number of } \\
\text { external nozzle }\end{array}$ \\
\hline 101325 & 570 & 0.8579 & 101325 & 328.15 & 0.9045 \\
\hline
\end{tabular}

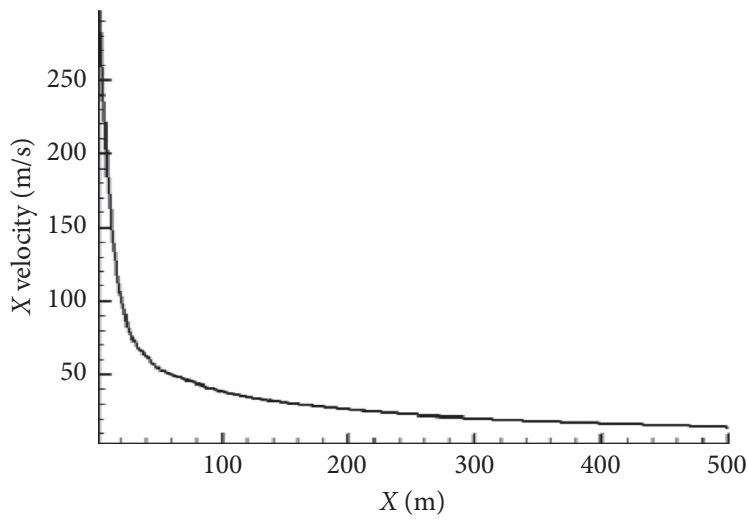

FIGURE 12: Velocity distribution curve of the A320 jet.

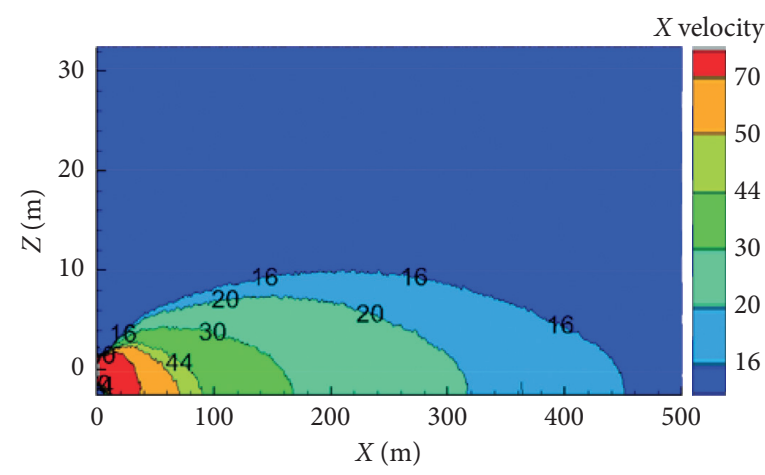

FIGURE 13: Velocity distribution cloud map of the XZ section of the A320 jet. 


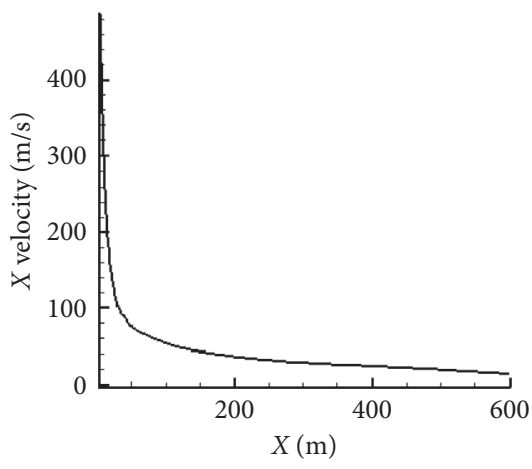

(a)

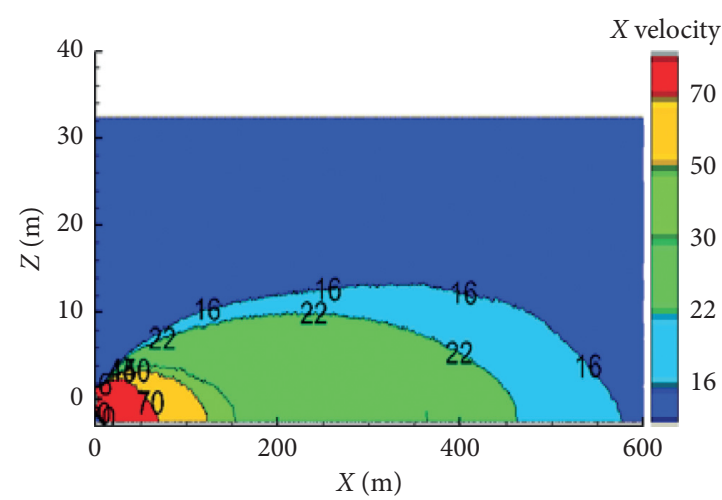

(b)

FIgURE 14: B777 jet velocity distribution curve and XZ section velocity distribution cloud map.

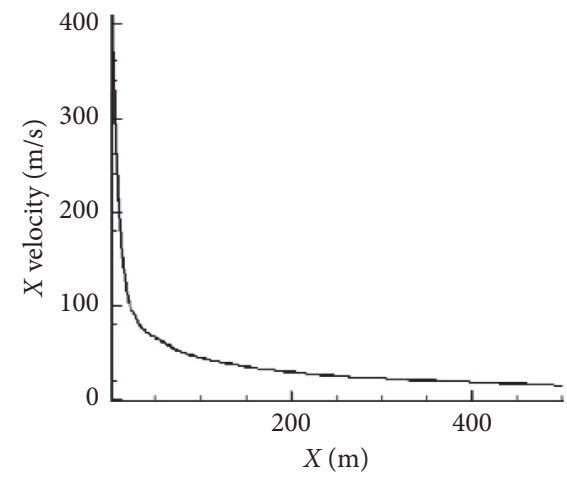

(a)

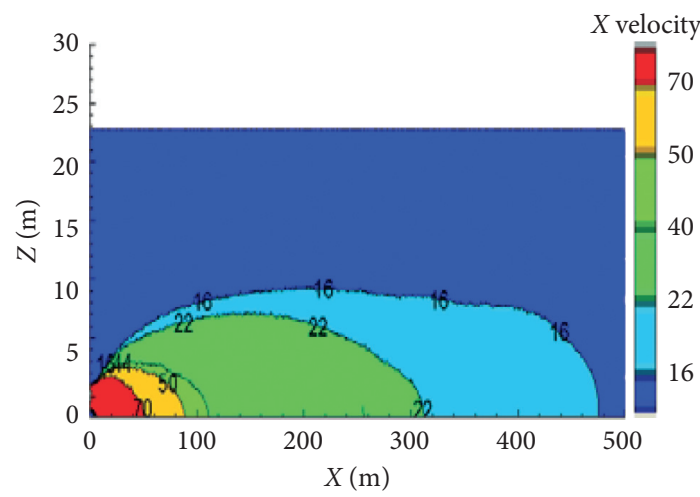

(b)

FIGURE 15: B737-800 jet velocity distribution curve and XZ section velocity distribution cloud map.

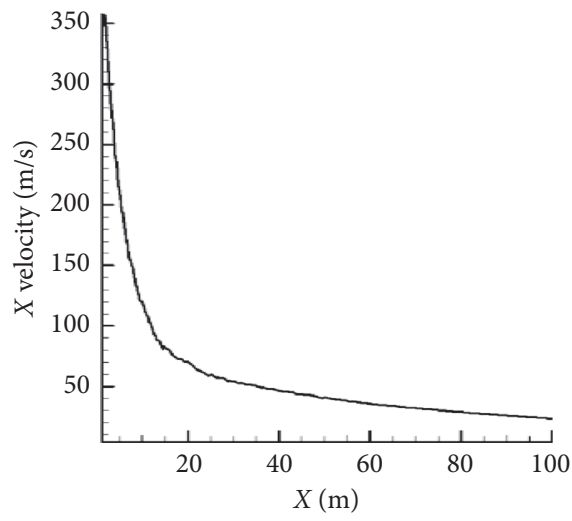

(a)

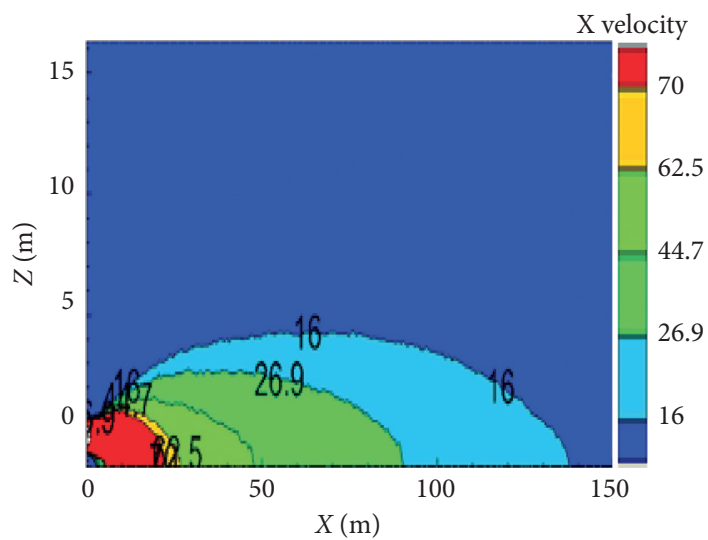

(b)

FIGURE 16: CRJ900 jet velocity distribution curve and $X Z$ section velocity distribution cloud map.

TABLe 5: Influence distance of a typical jet blast.

\begin{tabular}{lccc}
\hline Aircraft type & Influence distance of $44 \mathrm{~m} / \mathrm{s}$ & Influence distance of $22 \mathrm{~m} / \mathrm{s}$ & Influence distance of $16 \mathrm{~m} / \mathrm{s}$ \\
B777 & $153 \mathrm{~m}$ & $462 \mathrm{~m}$ & $576 \mathrm{~m}$ \\
A320 & $90 \mathrm{~m}$ & $296 \mathrm{~m}$ & $451 \mathrm{~m}$ \\
B737-800 & $86 \mathrm{~m}$ & $312 \mathrm{~m}$ & $475 \mathrm{~m}$ \\
CRJ900 & $47 \mathrm{~m}$ & $125 \mathrm{~m}$ & $157 \mathrm{~m}$ \\
\hline
\end{tabular}


In summary, the influence distances of the B777, A320, B737-800, and CRJ900 jet blast at speeds of $44 \mathrm{~m} / \mathrm{s}, 22 \mathrm{~m} / \mathrm{s}$, and $16 \mathrm{~m} / \mathrm{s}$ are given in Table 5 .

\section{Conclusion}

Based on the safe operation of the taxi-behind mode, the influence distance of jet flow in aircraft engine under the taxi-behind mode is numerically simulated. Based on the SA turbulence model, the DES-SA hybrid method is constructed. Cold spray and hot spray conditions are calculated using a nozzle model without considering the internal flow of the engine, and the state of the engine's actual working condition is determined. According to the selection of four presently used typical aircraft models, the jet effect of the engine is numerically simulated, and the influence distance of the jet blast is determined.

(1) The numerical simulation results for two jet types under cold and hot spray conditions indicate the following: whether the coning channel is heated or not, the speed at the axis of the engine nozzle decreases with the increase of the nozzle distance, and the velocity decreases rapidly within $100 \mathrm{~m}$ of the engine nozzle and decreases slowly beyond $100 \mathrm{~m}$. Compared with the unheated condition, the inner channel heating condition is more consistent with the actual working state of the engine.

(2) The numerical simulation results for a typical jet engine effect show the following: medium-duty and heavy-duty aircrafts typically use a more powerful engine with more horsepower than a light-duty aircraft. When the jet speed is $16 \mathrm{~m} / \mathrm{s}$, the influence distance is far greater than $300 \mathrm{~m}$. When the jet velocity speed is $16 \mathrm{~m} / \mathrm{s}$, the impact distance of the light-duty aircraft is much less than $300 \mathrm{~m}$. In the follow-up work of this study, a typical aircraft type will be selected to carry out the real engine jet effect experiment at the airport, and the corresponding parameters of the jet blast will be collected. The experimental data obtained will further verify the numerical simulation results in this study.

\section{Data Availability}

The data on engine parameters of typical aircrafts are derived from https://www.prattwhitney.com $\sim \sim \sim \sim \sim \sim \sim$ $\hat{\sim \hat{\sim}} \hat{\sim} \sim \sim \sim \sim \sim \sim \sim \sim \sim \mathrm{amp}$ and https://http://www. cfmaeroengines.com.

\section{Disclosure}

The funders had no role in the design of the study; in the collection, analyzes, or interpretation of data; in the writing of the manuscript; or in the decision to publish the results.

\section{Conflicts of Interest}

The authors declare that they have no conflicts of interest.

\section{Authors' Contributions}

X.H. and Y.C. conceptualized and supervised the study; X.H. developed methodology, performed formal analysis, collected data, and wrote the original draft; Y.M. developed software and investigated, edited, and reviewed the study; Y.C., X.H., D.H., and H.G. validated the study; Y.C. collected resources and fund; H.G. visualized the study; D.H. administered the project. All authors have read and agreed to the published version of the article.

\section{Acknowledgments}

The authors acknowledge the comments and suggestions from the editor and all the anonymous reviewers. This research was supported by CAAC Aviation Safety Capacity Building Fund Supported Project ( TM2018-3-1/2).

\section{References}

[1] Y. Chen, Y. Ma, and Hexin, "Analysis and research process of paired approach mode," Advances in Aeronautical Science and Engineering, vol. 11, no. 6, pp. 767-773, 2020.

[2] M. Yazdi, S. Daneshvar, and H. Setareh, "An extension to fuzzy developed failure mode and effects analysis (FDFMEA) application for aircraft landing system," Safety Science, vol. 98, pp. 113-123, 2017.

[3] M. Yazdi, S. Kabir, and M. Walker, "Uncertainty handling in fault tree based risk assessment: state of the art and future perspectives," Process Safety and Environmental Protection, vol. 131, pp. 89-104, 2019.

[4] J. Chen, P. Zhang, N. Zhou, and Y. Deng, "Application of detached-eddy simulation based on Spalart-Allmaras turbulence model," Journal of Beijing University of Aeronautics and Astronautics, no. 7, pp. 56-60, 2012.

[5] Y. Zhang, R. P. Rudis, F. Y. Wang et al., "Simulation of jet blast effect on landing aircraf," Air Traffic Control Quarterly, vol. 9, no. 3, pp. 211-227, 2016.

[6] S. Melber-Wilkending, "Aerodynamic analysis of jet-blast using CFD considering as example a Hangar and an AIRBUS A380 configuration," in New Results in Numerical and Experimental Fluid Mechanics V, H. J. Rath, C. Holze, H. J. Heinemann, R. Henke, and H. Hönlinger, Eds., Springer, Berlin, Germany, 2006.

[7] P. Slaboch, "An operational model for the prediction of jet blast," in Proceedings of the 50th AIAA Aerospace Sciences Meeting including the New Horizons Forum and Aerospace Exposition, Nashville, TN, USA, January 2012.

[8] K. Ishiko, A. Hashimoto, Y. Matsuo, and S. Watanabe, "Numerical examination of the effect of cross-wind on jet blast," in Proceedings of the 50th AIAA Aerospace Sciences Meeting including the New Horizons Forum and Aerospace Exposition, Nashville, TN, USA, January 2012.

[9] M. Bennett, S. M. Christie, A. Graham et al., "Abatement of an aircraft exhaust plume using aerodynamic baffles," Environmental Science \& Technology, vol. 47, no. 5, p. 2346, 2013.

[10] M. Taguchi, N. Semba, K. Mori et al., "Experimental study on jet blast at laboratory scale," Journal of Energy and Power Engineering, vol. 8, no. 8, pp. 1386-1392, 2014.

[11] L. P. Zhao, "Numerical simulation for high temperature and high pressure flow field of aircraft engine jet impingement," Ship Science and Technology, vol. 38, no. 1, pp. 145-149, 2016. 
[12] X.-Q. Liu and Y.-Z. Wu, "The computation of the lateral jet turbulence flow using DES method," Acta Aeronautica et Astronautica Sinica, vol. 25, no. 3, pp. 209-213, 2004.

[13] G. Xiang, S. Wang, and C. G. Soares, "Study on the motion of a freely falling horizontal cylinder into water using OpenFOAM," Ocean Engineering, vol. 196, p. 106811, 2020.

[14] X. Yu, G. Xiang, H. Collopy et al., "Trajectory prediction of a model rocket falling into the towing tank: experimental tests versus numerical simulations," Journal of Aerospace Engineering, vol. 33, no. 5, p. 04020056, 2020.

[15] G. Xiang and C. Guedes Soares, "Incorporating irregular nonlinear waves in simulation of dropped cylindrical objects," Proceedings of the Institution of Mechanical Engineers, Part M: Journal of Engineering for the Maritime Environment, vol. 234, no. 1, pp. 272-283, 2020.

[16] H. Li and C. G. Soares, "Reliability analysis of floating offshore wind turbines support structure using hierarchical Bayesian network," in Proceedings of the 29th European Safety and Reliability Conference, pp. 2489-2495, Hannover, Germany, September 2019.

[17] H. Li, H.-Z. Huang, Y.-F. Li, J. Zhou, and J. Mi, "Physics of failure-based reliability prediction of turbine blades using multi-source information fusion," Applied Soft Computing, vol. 72, pp. 624-635, 2018.

[18] G. Xiang and C. Guedes Soares, "Improved dynamical modelling of freely falling underwater cylinder based on CFD," Ocean Engineering, vol. 211, p. 107538, 2020.

[19] Z. Yang, "Large-eddy simulation: past, present and the future," Chinese Journal of Aeronautics, vol. 28, no. 1, pp. 11-24, 2015.

[20] X. Liu, W. Huang, and H. Li, "The simulation of separated exhaust jet noise," Science Technology and Engineering, vol. 19, no. 22, pp. 338-342, 2019.

[21] Z. Zhu, X. Cheng, and H. Pan, "Large eddy simulation of supersonic jet mixing flow," Journal of Aerospace Power, vol. 34, no. 1, pp. 210-216, 2019.

[22] Y. Sun, Separation Flow Study Based on RANS Method and RANS/LES Hybrid Method, Huazhong University of Science, Wuhan, China, 2018.

[23] G. Xiang, X. Li, X. Yu, Y. Luo, and Y. Cao, "Motion dynamics of dropped cylindrical objects in flows after water entry," Ocean Engineering, vol. 173, pp. 659-671, 2017.

[24] G. Xiang, L. Birk, X. Yu, and H. Lu, "Numerical study on the trajectory of dropped cylindrical objects," Ocean Engineering, vol. 130, pp. 1-9, 2017.

[25] H. Tan, The Numerical Simulation of Frigate Surface Flow Field with DES Method, Nanjing University of Aeronautics and Astronautics, Nanjing, China, 2017.

[26] L. Davidson and S. Dahlström, "Hybrid LES-RANS: an approach to make LES applicable at high Reynolds number," International Journal of Computational Fluid Dynamics, vol. 19, no. 6, pp. 415-427, 2005.

[27] M. A. Pakhomov and V. I. Terekhov, "RANS modeling of flow structure and turbulent heat transfer in pulsed gas-droplet mist jet impingement," International Journal of Thermal Sciences, vol. 100, no. 2, pp. 284-297, 2016.

[28] X. Liu, Y. Wu, and K. Chen, "Computation of lateral turbulent jets using m-SST DES model I," Acta Mechanica Sinica, no. 4, pp. 401-406, 2004.

[29] J. F. Dietiker and K. Hoffmann, "Detached-eddy-simulation of supersonic jet flows," in Proceedings of the 6th AIAA Aviation Technology, Integration and Operations Conference (ATIO), Wichita, KS, USA, September 2006.
[30] Z. Gong, J. Li, J. Shan, and H. Zhang, "Numerical investigation of powered jet effects by RANS/LES hybrid methods," Xibei Gongye Daxue Xuebao/Journal of Northwestern Polytechnical University, vol. 37, no. 3, pp. 565-571, 2019.

[31] F. D. Gao, D. X. Wang, H. D. Wang et al., "Numerical analysis and verification of the gas jet from aircraft engines impacting a jet blast deflector," Chinese Journal of Mechanical Engineering, vol. 31, no. 5, pp. 127-137, 2018.

[32] K. Yue, L. Cheng, H. Liu, and Y. Wang, "Analysis of jet blast impact of embarked aircraft on deck takeoff zone," Aerospace Science and Technology, vol. 45, no. 5, pp. 60-66, 2015.

[33] N. A. Buchmann, C. Atkinson, and J. Soria, "Ultra-high-speed tomographic digital holographic velocimetry in supersonic particle-laden jet flows," Measurement Science \& Technology, vol. 24, no. 2, pp. 827-837, 2012.

[34] D. R. Brooks, T. Ecker, K. T. Lowe et al., "Experimental Reynolds stress spectra in hot supersonic round jets," in Proceedings of the 52nd Aerospace Sciences Meeting, pp. 1227-1245, National Harbor, MD, USA, January, 2014.

[35] H. Li, A. P. Teixeira, and C. Guedes Soares, "A two-stage failure mode and effect analysis of offshore wind turbines," Renewable Energy, vol. 162, pp. 1438-1461, 2020.

[36] H. Li, C. Guedes Soares, and H.-Z. Huang, "Reliability analysis of a floating offshore wind turbine using Bayesian Networks," Ocean Engineering, vol. 217, p. 107827, 2020.

[37] H. Li, H. Diaz, and C. G. Soares, "A developed failure mode and effect analysis for floating offshore wind turbine support structures," Renewable Energy, vol. 164, pp. 133-145, 2020.

[38] Y. Chen and X. Zhang, "A preliminary study on schemeson crossing runway behind takeoff aircraft at the chongqing Jiangbei airport," Journal of Civil Aviation, vol. 2, no. 4, pp. 25-30, 2018.

[39] M. Yazdi and S. Kabir, "A fuzzy Bayesian network approach for risk analysis in process industries," Process Safety and Environmental Protection, vol. 111, pp. 507-519, 2017.

[40] M. Yazdi, F. Nikfar, and M. Nasrabadi, "Failure probability analysis by employing fuzzy fault tree analysis," International Journal of System Assurance Engineering and Management, vol. 8, no. 2, pp. 1177-1193, 2017.

[41] R. Paoli, L. Nybelen, J. Picot et al., "Effects of jet/vortex interaction on contrail formation in supersaturated conditions," Physics of Fluids, vol. 25, no. 5, pp. 25-30, 2013.

[42] M. Yazdi, A. Nedjati, E. Zarei, and R. Abbassi, "A novel extension of DEMATEL approach for probabilistic safety analysis in process systems," Safety Science, vol. 121, pp. 119-136, 2020.

[43] P. R. Spalart, "Detached-eddy simulation," Annual Review of Fluid Mechanics, vol. 41, no. 41, pp. 181-202, 2009.

[44] ANSYS Inc, ANSYS FLUENT User's Guide, ANSYS Inc, Shanghai, China, 2011.

[45] M. Shives and C. Crawford, "Adapted two-equation turbulence closures for actuator disk RANS simulations of wind \& tidal turbine wakes," Renewable Energy, vol. 92, pp. 273-292, 2016.

[46] A. Lopez, W. Nicholls, M. T. Stickland, and W. M. Dempster, "CFD study of jet impingement test erosion using Ansys fluent ${ }^{\circledR}$ and OpenFOAM ${ }^{\circledR}$," Computer Physics Communications, vol. 197, no. 6, pp. 88-95, 2015. 